\title{
NEW CHARACTERIZATIONS OF PSEUDO-FROBENIUS RINGS AND A GENERALIZATION OF THE FGF CONJECTURE
}

\author{
PEDRO A. GUIL ASENSIO, SERAP SAHINKAYA, AND ASHISH K. SRIVASTAVA \\ Dedicated to Alberto Facchini on his 60th birthday
}

\begin{abstract}
We provide new characterizations of pseudo-Frobenius and quasiFrobenius rings in terms of tight modules. In the process, we also provide fresh perspectives on FGF and CF conjectures. In particular, we propose new natural extensions of these conjectures which connect them with the classical theory of PF rings. Our techniques are mainly based on set-theoretic counting arguments initiated by Osofsky. Several corollaries and examples to illustrate their applications are given.
\end{abstract}

\section{INTRODUCTION.}

A ring $R$ is called right pseudo-Frobenius ( $\mathrm{PF}$, for short) when it is a right selfinjective right cogenerator ring. And a right $\mathrm{PF}$ ring is called quasi-Frobenius (QF, for short) when it is, moreover, right (and left) artinian. The origin of these rings can be drawn back to extensions of the concept of Frobenius algebras associated to the modular representations of finite groups (see e.g. [7]).

It is well known that a two-sided PF ring establishes a perfect duality in the sense of [23, Chapter 12, pages 307-308] and that a left and right cogenerator ring (in particular, a commutative cogenerator ring) is both-sided PF. The main reason why left and right cogenerator rings induce a perfect duality is that they are bothsided finitely cogenerated, that is, their left and right socles are finitely generated and essential in the ring (see e.g. [25, Theorem 19.18]). One sided PF rings were introduced and studied independently by Azumaya 2], Osofsky 28, and Utumi 33]. It is known that a right PF ring does not need to be left PF [8]. But a deep theorem of Osofsky showed that right PF rings still enjoy the properties of being semiperfect and having finitely generated essential right socle [28, Theorem 1].

On the other hand, it is easy to check that a ring in which any right module embeds in a free module is QF. This fact suggested Faith to conjecture in 9 that a ring is QF provided that any finitely generated right module embeds in a free module, thus extending an older question of Levy for commutative rings. And more generally, it is conjectured that a ring in which every cyclic right module embeds in a free module is right artinian. Rings satisfying that every cyclic (resp., finitely generated) right module embeds in a free module are usually called in the literature right $\mathrm{CF}$ (resp., right FGF) rings. And the question of whether any right CF (resp.,

2010 Mathematics Subject Classification. 16D40, 16D80, 16L60.

Key words and phrases. PF rings, QF rings, tight modules.

The first author has been partially supported by the DGI (MTM2010-20940-C02-02) and by the Excellence Research Groups Program of the Séneca Foundation of the Region of Murcia. Part of the sources of both institutions come from the FEDER funds of the European Union. 
right FGF) ring is right artinian (resp., QF) is nowadays known as the $\mathrm{CF}$ (resp., FGF) conjecture. Both conjectures are still open, whereas it is known that the CF conjecture implies the FGF conjecture and that they are true under many different additional hypothesis (see e.g. 9, 11, 13, 14, 17, 22]). Note that every right FGF ring is a right $\mathrm{CF}$ ring, but the converse is not true. Björk 4 gave an example of a right $\mathrm{CF}$ ring which is not right $\mathrm{FGF}$.

Probably the most promising partial positive results to the CF and FGF conjectures are based on using the set theoretical counting techniques developed by Osofsky in her proof that a right PF ring has finitely generated essential socle. This approach to the conjecture was initiated independently by Björk [5] and Tolskaya [32] who proved that every right self-injective right $\mathrm{CF}$ ring is right artinian. And it culminated in 11, where the authors proved that every ring in which any cyclic (resp., finitely generated) right module essentially embeds in a projective module is right artinian (resp., QF). They also proved in [12 that a right CF and right extending ring has finitely generated essential socle. In particular, any right cogenerator right extending ring is right PF. Note that a ring is called right extending (or right CS) if every right ideal is essential in a direct summand of the ring.

All the above results suggest that there might exist a deep relation between the characterization obtained by Osofsky of right $\mathrm{PF}$ rings and the $\mathrm{CF}$ and FGF conjectures. But surprisingly, it seems that there has not been any attempt in the literature of connecting both situations. The main purpose of the present paper is to highlight these connections, which allows us to obtain new non-trivial characterizations of right $\mathrm{PF}$ rings, as well as new partial positive answers to the $\mathrm{CF}$ and FGF conjectures.

Our approach is based on the notion of tight rings. Tight rings and modules were introduced by Golan and López-Permouth in [16] in order to study QI-filters and they have been later studied in [19, 21] in connection with weakly-injective modules. Recall that a ring $R$ is called right tight (resp., right $R$-tight) if every finitely generated (resp., every cyclic) submodule of its injective envelope $E\left(R_{R}\right)$ embeds in $R$. The definition of tightness is closely related to the notion of embedding of finitely generated or cyclic modules in free modules. Therefore, it seems natural to conjecture that they might play a role in the characterization of right PF rings, as well as in answering the CF and FGF conjectures. Moreover, any right PF ring is trivially right tight and thus, they are the natural candidate to establish a link between both notions. And as a byproduct, one may adapt, exploit and extend different deep techniques, which have been developed in order to solve these conjectures, to get nontrivial new characterizations of PF and QF rings.

We begin by extending in Theorem 2.1 the techniques developed in 11]. This allows us to obtain as corollaries the main results of [11, 12. Next, we study in Theorem 2.5 and Theorem 2.6 when a right tight cogenerator ring has a finitely generated essential right socle. Both results are inspired by the above mentioned transfinite counting arguments introduced by Osofsky in [28] which, in turn, were based on an old result of Tarski on almost disjoint partitions of infinite sets [31. The obtained results allow us to establish the following conjecture:

Conjecture 1. Every right cogenerator right $R$-tight ring is right $\mathrm{PF}$. 
We finish this section by proving this conjecture under different additional conditions and exhibiting several corollaries and examples which illustrate the applications and limits of the developed theory.

We begin Section 3 by observing that the obtained results naturally lead to establish the following new conjecture that encompasses the different open questions and conjectures existing on the topic:

Conjecture 2. Every right Kasch generalized right $(R$-)tight ring has finitely generated and essential right socle.

Recall that a ring $R$ is called right Kasch when it cogenerates all simple right modules. In particular, any right cogenerator ring is right Kasch. And $R$ is called generalized right $(R$-)tight if every finitely generated (resp., cyclic) submodule of $E\left(R_{R}\right)$ embeds in a free module. It may be noted here that a positive solution to Conjecture 2 would imply affirmative answers to both Conjecture 1 and the CF and FGF conjectures. Note also that Osofsky's characterization of right PF rings can be seen as a particular solution to this conjecture when the ring is assumed to be right self-injective.

We dedicate the rest of the paper to show that our new conjecture is satisfied when we assume the different additional conditions under which the CF and FGF conjectures are known to be true. This shows that this conjecture naturally extends the CF and FGF conjectures, and it connects them to Osofsky's work on PF rings. Moreover, as a byproduct of these results, we obtain new partial positive answers to the $\mathrm{CF}$ and FGF conjectures.

Throughout this paper, all rings $R$ will be associative and with identity, and Mod- $R$ will denote the category of right $R$-modules. We will use the notation $M_{R}$ to stress the right $R$-module structure of a module $M$, when necessary. We will denote by $J(R)$, the Jacobson radical of a ring $R$ and by $Z\left(R_{R}\right)$, the singular right ideal of $R$ consisting of those elements of $R$ which have essential right annihilator. We refer to [1, 22, 25, 27, 30] for all undefined notions used in the text.

\section{NeW Characterizations of PF Rings.}

We begin by proving several extensions of [28, Theorem 1] which will be used in our characterization of right $\mathrm{PF}$ rings. As a consequence, we will also deduce the main results of [11, 12]. Recall that a ring $R$ is called right Kasch if every simple right module embeds in $R$.

Theorem 2.1. Let $R$ be a right Kasch ring such that each cyclic submodule of the injective envelope $E\left(R_{R}\right)$ embeds in a free module. Assume that every direct summand of $E\left(R_{R}\right)$ contains an essential projective module $P$ such that $P /(P$. $\left.Z\left(R_{R}\right)\right)$ is finitely generated. Then $R_{R}$ has a finitely generated essential socle.

Proof. Let $E=E\left(R_{R}\right)$. As in [11, Lemma 2.4], we first show that if $S=\operatorname{End}\left(E_{R}\right)$ and $\left\{C_{k}\right\}_{k \in K}$ is an idempotent-orthogonal family of simple right $S / J$-modules (with $J=J(S)$ ), then there exists an injective mapping from index set $K$ to the set $\Omega(R)$ of isomorphism classes of simple right $R$-modules.

Since idempotents of $S / J$ lift modulo $J$, there exist idempotents $\left\{e_{k}\right\}_{k \in K}$ of $S$ such that $C_{k} e_{k} \neq 0$ for any $k \in K$ and either $C_{j} e_{k}=0$ or $C_{k} e_{j}=0$ for $k \neq j$. Let $c_{k} \in C_{k}$ be such that $c_{k} e_{k} \neq 0$ for each $k \in K$, and let $p_{k}: S_{S} \rightarrow C_{k}$ be the homomorphism defined as $p_{k}(1)=c_{k} e_{k}$. If $e_{k^{*}}=\operatorname{Hom}_{R}\left(E, e_{k}\right)$ is the 
endomorphism of $S_{S}$ given by left multiplication with $e_{k}$, we have $\left(p_{k} \circ e_{k^{*}}\right)(1)=$ $c_{k} e_{k}^{2}=c_{k} e_{k}=p_{k}(1)$, and so $p_{k} \circ e_{k^{*}}=p_{k}$. Thus it follows that

$$
\left(p_{k} \otimes_{S} E\right) \circ e_{k}=\left(p_{k} \otimes_{S} E\right) \circ\left(e_{k^{*}} \otimes_{S} E\right)=\left(p_{k} \circ e_{k^{*}}\right) \otimes_{S} E=p_{k} \otimes_{S} E .
$$

Set $E_{k}=\operatorname{Im}\left(e_{k}\right)$. We have by hypothesis that $E_{k}=E\left(P_{k}\right)$ with $P_{k}$, a projective module such that $P_{k} /\left(P_{k} \cdot Z\left(R_{R}\right)\right)$ is finitely generated. By hypothesis, each finitely generated submodule of $E_{k}$ embeds in a free module. Then $\left(p_{k} \otimes_{S} E\right)\left(P_{k}\right) \neq 0$ by [10, Proposition 1.3]. Let $h_{k}: P_{k} \rightarrow E_{k}, i_{k}: E_{k} \rightarrow E$, and $t_{k}=i_{k} \circ h_{k}: P_{k} \rightarrow E$ be the inclusions, and set $L_{k}:=\operatorname{Im}\left(\left(p_{k} \otimes_{S} E\right) \circ t_{k}\right)$, with canonical projection $q_{k}: P_{k} \rightarrow L_{k}$ and inclusion $w_{k}: L_{k} \rightarrow C_{k} \otimes_{S} E$. Note that $C_{k}$ is a right $S / J-$ module and thus, $L_{k}$ is a right $R / Z\left(R_{R}\right)$-module. Therefore, $\left(p_{k} \otimes_{S} E\right) \circ t_{k}$ factors through $P_{k} /\left(P_{k} \cdot Z\left(R_{R}\right)\right) \cong P_{k} \otimes_{R}\left(R / Z\left(R_{R}\right)\right)$ and so, $L_{k}$ is finitely generated. This means that we can choose for each $k \in K$, a simple quotient $U_{k}$ of $L_{k}$ with canonical projection $\pi_{k}: L_{k} \rightarrow U_{k}$. We define a map from index set $K$ to the set $\Omega(R)$ by assigning $k \mapsto\left[U_{k}\right]$, where $\left[U_{k}\right]$ denotes the isomorphism class of the simple module $U_{k}$. It may be checked that this map is injective.

Now, since $R_{R}$ cogenerates the simple modules by hypothesis, we have, as shown in [11, that $|\Omega(R)| \leq|C(R)|$ where $C(R)$ denotes a set of representatives of the isomorphism classes of simple submodules of $R$. Let $\mathcal{M}$ represent the set of isomorphism classes of minimal right ideals of $S / J$ and assume $|\Omega(R)|=n$. We claim that $|\mathcal{M}|=n$. Let $C_{1}, \ldots, C_{r}$ be a set of representatives of the elements of $\mathcal{M}$. Suppose that there exists a simple right $S$-module $C=C_{r+1}$ which is not isomorphic to any of the $C_{i}$, for $1 \leq i \leq r$. There exist idempotent elements $e_{1}, e_{2}, \ldots, e_{r} \in S$ such that, if $\bar{e}_{i}=e_{i}+J$, then $C_{i}=\bar{e}_{i}(S / J)$ for each $1 \leq i \leq r$. Since $\bar{e}_{i}(S / J) \bar{e}_{j}=\operatorname{Hom}_{S / J}\left(\bar{e}_{j}(S / J), \bar{e}_{i}(S / J)\right)$, we have $\bar{e}_{i}(S / J) \bar{e}_{j}=0$ for $i, j \leq r$, $i \neq j$ and $\bar{e}_{i}(S / J) \bar{e}_{i} \neq 0$ for all $i=1, \ldots, r$. Thus the family $\left\{C_{i}\right\}, i=1, \ldots, r+1$ is an idempotent-orthogonal family of simple right $S / J$-modules with respect to the idempotents $\left\{\bar{e}_{1}, \ldots, \bar{e}_{r}, 1\right\}$. We have then $r+1 \leq n$, a contradiction that shows that the simple module $C$ cannot exist, and hence that $S / J$ is a semisimple artinian ring. Therefore $S$ is a semiperfect ring and $E_{R}$ is a finite-dimensional module. Thus $E_{R}$ is a finite direct sum of indecomposable submodules. From the preceding argument it also follows that $r \leq n$ and hence that $r=n$. Since there exists a bijection between the set of isomorphism classes of indecomposable direct summands of $E_{R}$ and the set $\mathcal{M}$ of isomorphism classes of minimal right ideals of $S / J$, the number of isomorphism classes of indecomposable direct summands of $E_{R}$ is exactly $n$, and so each of them is an injective envelope of a simple right $R$-module, so that $E_{R}$, and hence $R_{R}$ has finite essential socle.

As a consequence, we have the following.

Corollary 2.2. 11, Corollary 3.3, Corollary 3.5] Let $R$ be a ring. If every cyclic right $R$-module essentially embeds in a projective module, then $R$ is right artinian.

If moreover, every finitely generated right $R$-module essentially embeds in a free module, then $R$ is $Q F$.

Proof. Let us first show that $\operatorname{Soc}\left(R_{R}\right)$ is finitely generated and essential. In order to apply Theorem 2.1, we only need to show that any direct summand of $E=E\left(R_{R}\right)$ contains an essential projective submodule $P$ such that $P /\left(P \cdot Z\left(R_{R}\right)\right)$ is a finitely generated right module.

Let $E^{\prime}$ be a nonzero direct summand of $E$. As $R_{R}$ is essential in $E, E^{\prime}$ contains an essential cyclic module $x R$. By hypothesis, there exists an essential monomorphism 
$u: x R \rightarrow P$, for some projective module $P$. And this essential monomorphism extends by injectivity to a monomorphism $v: P \rightarrow E^{\prime}$. Therefore, $E^{\prime}$ contains the essential projective submodule $P$.

Let us now check that $P /\left(P \cdot Z\left(R_{R}\right)\right)$ is finitely generated. As $P$ is projective, it is a direct summand of a free module, say $R^{(I)}$. Let $w: P \rightarrow R^{(I)}$ and $p: R^{(I)} \rightarrow P$ be the canonical injection and projection. Now, as $x R$ is cyclic, there exists a finite subset $I^{\prime}$ of $I$ such that $\left.w \circ u\right|_{x R} \subseteq R^{\left(I^{\prime}\right)}$. Let $\pi: R^{(I)} \rightarrow R^{\left(I^{\prime}\right)}$ and $i: R^{\left(I^{\prime}\right)} \rightarrow R^{(I)}$ be the projection and injection, respectively. Then $\left.p \circ w\right|_{x R}-\left.p \circ i \circ \pi \circ w\right|_{x R}=0$ and therefore, as $x R$ is essential in $E^{\prime}$, this means that $\operatorname{Im}(p \circ w-p \circ i \circ \pi \circ w) \in P \cdot Z\left(R_{R}\right)$. Therefore, as $p \circ w=1_{P}$, we deduce that

$$
P /\left(P \cdot Z\left(R_{R}\right)\right)=\left(\operatorname{Im}(\pi \circ i \circ \pi \circ w)+P \cdot Z\left(R_{R}\right)\right) /\left(P \cdot Z\left(R_{R}\right)\right)
$$

and therefore, it is finitely generated as it is a homomorphic image of $R^{\left(I^{\prime}\right)}$. The proof now follows from the arguments used in [11, Corollary 3.3, Corollary 3.5].

Recall that a ring $R$ is called right extending (or right CS) if every right ideal essentially embeds in a direct summand of $R$.

Corollary 2.3. 12, Corollary 2.7] Let $R$ be a right Kasch ring. If $R_{R}$ is extending, then it has finitely generated essential socle.

Proof. If $R_{R}$ is extending, then clearly any direct summand of $E\left(R_{R}\right)$ contains an essential direct summand of $R$. So the result follows from Theorem 2.1

Thus if $R$ is right extending and each cyclic right $R$-module embeds in a free module, then each cyclic right $R$-module has finitely generated essential socle and consequently the ring is right artinian.

Corollary 2.4. [12, Corollary 2.9] If $R$ is a right extending ring, then both $C F$ and $F G F$ conjectures hold for $R$.

The next theorem will be essential for obtaining our new characterizations of right $\mathrm{PF}$ rings. Its proof is based on transfinite counting arguments inspired by [28, Theorem 1] and [14, Theorem 6]. We will say that a ring $R$ has completely nil Jacobson radical if for any two-sided ideal $N$ of $R$, any element in the Jacobson radical of $R / N$ is nilpotent.

Theorem 2.5. Let $R$ be a right cogenerator right $R$-tight ring. If $R / Z\left(R_{R}\right)$ has completely nil Jacobson radical, then $\operatorname{Soc}\left(R_{R}\right)$ is finitely generated and essential in $R_{R}$.

Proof. Let us first fix our notation. We will denote the injective envelope of $R_{R}$ by $E=E\left(R_{R}\right)$ with inclusion $u: R_{R} \rightarrow E$. Let us set $S=\operatorname{End}_{R}(E)$ and $J=J(S)$, where $J(S)$ is the Jacobson radical of $S$. It is shown in [14, Lemma 1] that there exists a homomorphism of rings $\Phi: R \rightarrow S / J$ which assigns any element $r \in R$ to the element $s_{r}+J$, where $s_{r}$ is an endomorphism of $E$ which extends the left multiplication by $r$. The kernel of $\Phi$ is the singular ideal $Z\left(R_{R}\right)$ of $R_{R}$. Therefore, we get an injective homomorphism of rings $\Psi: R / Z\left(R_{R}\right) \rightarrow S / J$ induced by $\Phi$.

Let us now show that $\operatorname{Soc}\left(R_{R}\right)$ is finitely generated and essential in $R_{R}$. We are going to prove it in three steps, as in [14, Theorem 6].

Step 1. We claim that $\operatorname{Soc}\left(R_{R}\right)$ contains only finitely many homogeneous components. 
We will assume that $\operatorname{Soc}\left(R_{R}\right)$ has infinitely many homogeneous components and we will try to reach a contradiction. Let $\left\{C_{i}\right\}_{i \in I}$ be a representative set of the isomorphism classes of simple modules in $\operatorname{Soc}\left(R_{R}\right)$. By Tarski's Lemma 31. (see also [28]), there exists a a family $\mathcal{K}$ of almost disjoint subsets of $I$ such that $|\mathcal{K}| \supsetneqq|I|$ and $I$ is the union of the sets in this family. In other words there exists a family $\mathcal{K}$ of subsets of $I$ satisfying that:

- $I=\cup_{K \in \mathcal{K} K} K$ and $|\mathcal{K}| \supsetneqq|I|$.

- $|K|$ is infinite for any $K \in \mathcal{K}$.

- $|K|=\left|K^{\prime}\right| \supsetneqq\left|K \cap K^{\prime}\right|$ for any $K, K^{\prime} \in \mathcal{K}$ with $K \neq K^{\prime}$.

We know that there exists an injective map from the index set I to the family of isomorphism classes of minimal right ideals of $S / J$ [14, Lemma 3]. This map assigns any element $i \in I$ to the minimal right ideal $e_{i} S / e_{i} J$ of $S / J$, where $e_{i} \in S$ is an idempotent such that $e_{i} E=E\left(C_{i}\right)$.

Let us take any subset of $I$, say $A$, and set

$$
X_{A}=E\left(\sum\left\{D \leq S / J_{S / J} \mid D \cong e_{C_{i}} S / e_{C_{i}} J \text { for some } i \in A\right\}\right)
$$

Since $X_{A}$ is a direct summand of $E$, there exists an idempotent $e_{A} \in S$ such that $X_{A}=e_{A} E$. We know by [14, Lemma 4] that $e_{A}+J$ is a central idempotent in $S / J$. In particular, $\left(\left(1-e_{I}\right) S+J\right) / J$ is a two sided ideal of $S / J$ when $A=I$. For simplicity, $\left(\left(1-e_{I}\right) S+J\right) / J$ will be denoted by $\mathcal{N}_{I} / J$. Since $\mathcal{N}_{I} / J$ is a two-sided ideal, its inverse image $\Psi^{-1}\left(\mathcal{N}_{\mathcal{I}} / J\right)$ is a two sided ideal of $R / Z\left(R_{R}\right)$ and we will denote it by $\mathcal{M}_{I} / Z\left(R_{R}\right)$.

Let $\aleph=|K|$ and let us set

$$
\mathcal{N} / J=\mathcal{N}_{I} / J+\sum\left\{\left(e_{A} S+J\right) / J \mid A \subseteq I \text { with }|A| \varsubsetneqq \aleph\right\}
$$

and call $\mathcal{M} / Z\left(R_{R}\right)=\Psi^{-1}(\mathcal{N} / J)$. By [14, Lemma 7], we know that $\left\{e_{K}+\mathcal{N} \mid K \in\right.$ $\mathcal{K}\}$ is an orthogonal family of nonzero central idempotents in $S / \mathcal{N}$.

Let $u$ be the inclusion of $R_{R}$ in its injective envelope $E$ and call $x_{K}=e_{K} \circ u(1) \in$ $E$. Then $e_{K} \circ u$ factors as

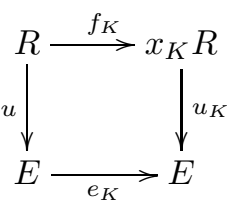

where $f_{K}$ is an epimorphism and $u_{K}$, a monomorphism. There exists a monomorphism $\alpha_{K}: x_{K} R \rightarrow R$ by our assumption that any cyclic submodule of $E\left(R_{R}\right)$ embeds in $R$. By injectivity, $\alpha_{K}$ extends to an $s_{K}: E \rightarrow E$ such that $u \circ \alpha_{K}=$ $s_{K} \circ u_{K}$. Again, as $\left.s_{K}\right|_{E\left(x_{K} R\right)}: E\left(x_{K} R\right) \rightarrow E$ is a monomorphism, there exists an $h_{K}: E \rightarrow E$ such that $h_{K} \circ s_{K} \circ e_{K}=e_{K}$.

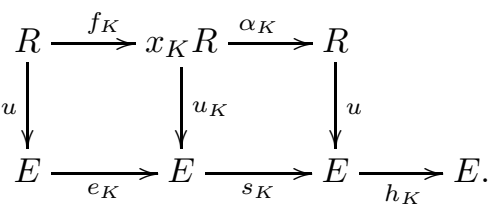

Call $r_{K}=s_{K} \circ e_{K} \circ u(1) \in R$. Our claim is that $r_{K}+\mathcal{M} \notin J(R / \mathcal{M})$. Assume otherwise that $r_{K}+\mathcal{M} \in J(R / \mathcal{M})$. As we are assuming that $J\left(R / Z\left(R_{R}\right)\right)$ is 
completely nil, we deduce that any element in $J(R / \mathcal{M})$ is nilpotent. Thus, there exists a natural number $m \geq 1$ such that $r_{K}^{m}+\mathcal{M}=0$ in $R / \mathcal{M}$. But then, $s_{K}^{m} \circ e_{K}+\mathcal{N}=\Phi\left(r_{K}^{m}+\mathcal{M}\right)=0$. Therefore, we get that

$$
\begin{aligned}
0 & =h_{K}^{m} \circ s_{K}^{m} \circ e_{K}+\mathcal{N}=h_{K}^{m-1} \circ\left(h_{K} \circ s_{K} \circ e_{K}\right) \circ s_{K}^{m-1}+\mathcal{N} \\
& =h_{K}^{m-1} \circ e_{K} \circ s_{K}^{m-1}+\mathcal{N}=\ldots=h_{K} \circ s_{K} \circ e_{K}+\mathcal{N}=e_{K}+\mathcal{N}
\end{aligned} .
$$

But it is a contradiction since $e_{K}$ does not belong $\mathcal{N}$ by construction.

As $r_{K}+\mathcal{M} \notin J(R / \mathcal{M})$, there exists a maximal right ideal $L_{K} / \mathcal{M}$ of $R / \mathcal{M}$ such that $r_{K}+\mathcal{M} \notin L_{K} / \mathcal{M}$. Thus, $R / L_{K}$ is a simple right $R$-module which satisfies $R / L_{K} \cdot\left(r_{K}+\mathcal{M}\right) \neq 0$.

We finally claim that $R / L_{K} \nsucceq R / L_{K^{\prime}}$ when $K \neq K^{\prime}$ with $K, K^{\prime} \in \mathcal{K}$. Assume that $\delta: R / L_{K} \rightarrow R / L_{K^{\prime}}$ is an isomorphism. We get that

$$
0 \neq \delta\left(r_{K}+L_{K}\right)=\delta\left(1+L_{K}\right) \cdot\left(r_{K}+\mathcal{M}\right) .
$$

In particular, $\delta\left(1+L_{K}\right) \neq 0$. On the other hand, $\delta\left(1+L_{K}\right)$ is a generator of $R / L_{K^{\prime}}$, since it is simple. And we know that $\left(R / L_{K}^{\prime}\right) \cdot\left(r_{K^{\prime}}+\mathcal{M}\right) \neq 0$, which assures the existence of an $r \in R$ such that $0 \neq \delta\left(1+L_{K}\right) \cdot\left(r_{K} r r_{K^{\prime}}+\mathcal{M}\right)$. But then, $r_{K} r r_{K^{\prime}}+\mathcal{M} \neq 0$ and thus, $\Psi\left(\left(r_{K} r r_{K^{\prime}}+\mathcal{M}\right)\right) \neq 0$, by the injectivity of $\Psi$. We deduce that

$$
s_{K} \circ h_{K} \circ e_{K} \circ \Psi(r+\mathcal{M}) \circ s_{K^{\prime}} \circ h_{K^{\prime}} \circ e_{K^{\prime}}+\mathcal{N} \neq 0 .
$$

But both idempotents are central in $S / \mathcal{N}$ and so, $e_{K} \circ e_{K^{\prime}} \notin \mathcal{N}$. And this means that $K=K^{\prime}$, since otherwise $e_{K} \circ e_{K^{\prime}} \in \mathcal{N}$ by construction.

We have constructed then a family $\left\{R / L_{K}\right\}_{K \in \mathcal{K}}$ of non isomorphic simple right $R$-modules with $|\mathcal{K}| \gtreqless|I|$ isomorphism classes of right simple modules. This is a contradiction since we have assumed that $R_{R}$ has $|I|$ non isomorphic classes of simple right modules. Therefore, $\operatorname{Soc}\left(R_{R}\right)$ must have only finitely many homogeneous components.

Step 2. We claim that any homogeneous component of $\operatorname{Soc}\left(R_{R}\right)$ is finitely generated.

We know by Step 1 that there are only finitely many homogeneous components in $\operatorname{Soc}\left(R_{R}\right)$. Let $\left\{C_{1}, \ldots, C_{m}\right\}$ be a representative set of simple modules belonging to them. As $R_{R}$ is a cogenerator, there exist sets of orthogonal idempotents $\left\{r_{i} \in\right.$ $R \mid i=1, \ldots, m\}$ and $\left\{e_{i} \in E \mid i=1, \ldots, m\right\}$ such that $E\left(C_{i}\right)=r_{i} R=e_{i} E$ for each $i=1, \ldots, m$. In particular, this means that $\Psi\left(r_{i}+Z\left(R_{R}\right)\right)=e_{i}+J$. And $r_{i}+Z\left(R_{R}\right)$ does not belong to the Jacobson radical of $R / Z\left(R_{R}\right)$ for any $i=1, \ldots, m$, since they are idempotent. Moreover, $E\left(C_{i}\right)$ is the projective cover of a simple right module $D_{i}$ since they are indecomposable injective direct summands of $R_{R}$. Note also that, $D_{i} \not D_{j}$ when $i \neq j$ and $D_{i} \cdot r_{i} \neq 0$ for each $i \in I$, by construction.

Now assume that some homogeneous component is not finitely generated. Say that it is the homogeneous component associated to $C_{1}$. Repeating the arguments in Step 1 , but replacing $K$ by $A=\{i\}$ and $\mathcal{N}$ by $\mathcal{N}^{\prime}=\operatorname{Soc}\left(S / J_{S / J}\right)$, we may construct a central idempotent $e_{A}+J \in S / J$ such that $\left(e_{A} S+J\right) / J$ is the injective envelope of the homogeneous component corresponding to $e_{1} S / e_{1} J$ inside $S / J$. And we can find an element $r_{A}+\Psi^{-1}\left(\mathcal{N}^{\prime}\right)$ which does not belong to $J\left(R / \Psi^{-1}\left(\mathcal{N}^{\prime}\right)\right)$ and $\Psi\left(r_{A}+J\right)=h_{A} \circ s_{A} \circ \circ e_{A} \notin \operatorname{Soc}\left(S / J_{S / J}\right)$. Let us choose a maximal right ideal $L / \Phi^{-1}\left(\mathcal{N}^{\prime}\right)$ of $R / \Phi^{-1}\left(\mathcal{N}^{\prime}\right)$ satisfying that $r_{A}+L \neq 0$ in $R / \Phi^{-1}\left(\mathcal{N}^{\prime}\right)$. Note that this maximal right ideal does exist since $r_{A}+\Phi^{-1}\left(\mathcal{N}^{\prime}\right) \notin J\left(R / \Phi^{-1}\left(\mathcal{N}^{\prime}\right)\right)$. 
This means that, if we set $D=R / L$, this is a simple right $R$-module such that $D \cdot\left(r_{A}+R / \Phi^{-1}\left(\mathcal{N}^{\prime}\right)\right) \neq 0$.

We claim that $D$ is not isomorphic to $D_{i}$ for any $i=1, \ldots, m$. Assume on the contrary that $\delta: D \rightarrow D_{i}$ is an isomorphism for some $i=1, \ldots, m$. And fix a nonzero element $x \in D_{i}$ such that $x \cdot\left(r_{i}+Z\left(R_{R}\right)\right) \neq 0$. This means that $\delta(x) \cdot\left(r_{i}+Z\left(R_{R}\right)\right) \neq 0$ and so, it is a generator of $R / L$. Since $D \cdot\left(r_{A}+\Phi^{-1}\left(\mathcal{N}^{\prime}\right)\right) \neq$ 0 , there exists an $r \in R$ such that $x \cdot\left(r_{i} r r_{A}+J\right) \neq 0$ in $D$. And therefore, $r_{A} r r_{i} \notin \Phi^{-1}\left(\mathcal{N}^{\prime}\right)$, because $D$ is a right $R / \Phi^{-1}\left(\mathcal{N}^{\prime}\right)$-module. But then, $e_{i} \circ \Phi(r+$ $J) \circ h_{A} \circ s_{A} \circ e_{A}+J=\Phi\left(r_{i} r r_{A}+Z\left(R_{R}\right)\right) \notin \operatorname{Soc}\left(S / J_{S / J}\right)$. And this is a contradiction since $\left(e_{i}+J\right) / J \in \operatorname{Soc}(S / J)$ and $\operatorname{Soc}(S / J)$ is a two sided ideal.

We have shown that each homogeneous component of $\operatorname{Soc}\left(R_{R}\right)$ is finitely generated and so we proved that $\operatorname{Soc}\left(R_{R}\right)$ is finitely generated.

Step 3. We finally claim that $\operatorname{Soc}\left(R_{R}\right)$ is essential in $R_{R}$.

Repeating the arguments of Step 2, we may construct sets of orthogonal idempotents $\left\{r_{1}, \ldots, r_{m}\right\}$ and $\left\{e_{1}, \ldots, e_{m}\right\}$ in $R$ and $S$ associated to a representative family $\left\{C_{1}, \ldots, C_{m}\right\}$ of the isomorphism classes of the simple right ideals of $R$ such that $E\left(C_{i}\right)=e_{i} E=r_{i} R$ and $\Psi\left(r_{i}+Z\left(R_{R}\right)\right)=e_{i}+J$ for each $i=1, \ldots, m$. Let $D_{i}$ be a simple module such that $E\left(C_{i}\right)$ is a projective cover of $D_{i}$. We get that $D_{i} \cong D_{j}$ when $i \neq j$ and $D_{i} \cdot r_{i} \neq 0$ for each $i=1, \ldots, m$.

Assume that $\operatorname{Soc}\left(R_{R}\right)$ is not essential in $R_{R}$. This means that $E\left(\operatorname{Soc}\left(R_{R}\right)\right) \neq$ $E\left(R_{R}\right)$. Let $e_{I} \in S$ be the idempotent such that $E\left(\operatorname{Soc}\left(R_{R}\right)\right)=e_{I} E$. The arguments of Step 1 show that $e_{I}+J$ is central in $S / J$.

Repeating the arguments used in Step 2, but by replacing the idempotent $e_{A}$ by $1-e_{I}$ and the ideal $\mathcal{N}^{\prime}$ by $J$, we get an $r_{I} \in R$ such that $r_{I}+Z\left(R_{R}\right) \notin J\left(R / Z\left(R_{R}\right)\right)$ and elements $s_{I}, h_{I} \in S$ such that $h_{I} \circ s_{I} \circ\left(1-e_{I}\right) \notin J$ and $\Phi\left(r_{I}+Z\left(R_{R}\right)\right)=$ $s_{I} \circ\left(1-e_{I}\right)+J$. Therefore, there exists a maximal right ideal $L / Z\left(R_{R}\right)$ of $R / Z\left(R_{R}\right)$ such that $r_{I}+Z\left(R_{R}\right) \notin L / Z\left(R_{R}\right)$. In particular, if we call $D=R / L$, we get that $D \cdot\left(r_{I}+Z\left(R_{R}\right)\right) \neq 0$.

We claim that $D ¥ D_{i}$ for any $i=1, \ldots, m$. Let us assume on the contrary that $\delta: D \rightarrow D_{i}$ is an isomorphism and choose an $x \in D$ such that $x \cdot\left(r_{I}+Z\left(R_{R}\right)\right) \neq 0$. We then obtain that $\delta(x) \cdot\left(r_{I}+Z\left(R_{R}\right)\right) \neq 0$ as in Step 2. And thus, there exists an $r \in R$ satisfying that $\delta(x) \cdot\left(r_{I} r r_{i}+Z\left(R_{R}\right)\right) \neq 0$. Hence $r_{I} r r_{i}+Z\left(R_{R}\right) \neq 0$ and therefore $h_{I} \circ s_{I} \circ\left(1-e_{I}\right) \circ \Psi\left(r+Z\left(R_{R}\right)\right) \circ e_{i}+J \neq 0$ in $S / J$. And, as $\left(1-e_{I}\right)+J$ is central in $S / J$, we deduce that $\left(1-e_{I}\right) \circ e_{i}+J \neq 0$. But this is not possible because $e_{I} \cdot e_{i}=e_{i}$ by construction of $e_{I}$. So we get a contradiction which shows $\operatorname{Soc}\left(R_{R}\right)$ is essential in $R_{R}$.

We can now state our new characterizations of right PF rings.

Theorem 2.6. Let $R$ be a ring. Then the following conditions are equivalent:

(1) $R$ is right $P F$.

(2) $R_{R}$ is a cogenerator and every cyclic submodule of $E\left(R_{R}\right)$ essentially embeds in a projective module.

(3) $R$ is a right $(R-)$ tight cogenerator and $J\left(R / Z\left(R_{R}\right)\right)$ is completely nil.

(4) $R_{R}$ is tight, $R$ is semilocal, and the injective envelopes of simple right $R$ modules are finitely generated.

Proof. (1) $\Rightarrow(2)$. This is straightforward. 
$(1) \Rightarrow(3) . R$ is a right self-injective semiperfect ring. Therefore, $J(R)=Z\left(R_{R}\right)$ and $R / Z(J(R))$ is von Neumann regular. Thus, any ring which is a homomorphic image of $R / J(R)$ has zero Jacobson radical.

$(2)$ or $(3) \Rightarrow(4)$. By Theorem 2.1 and Theorem 2.5 , we get that $\operatorname{Soc}\left(R_{R}\right)$ is finitely generated and essential in $R$. In particular, there exists a finite number of isomorphism classes of simple modules, say $\left\{C_{1}, \ldots, C_{m}\right\}$. Moreover, as $R_{R}$ is a cogenerator, the injective envelopes of simple right $R$-modules are direct summands of $R$ and thus, they are projective and finitely generated. Since each $E\left(C_{i}\right)$ is projective, it is a local module and hence it is the projective cover of the simple module $E\left(C_{i}\right) / E\left(C_{i}\right) J(R)$. Note that $E\left(C_{i}\right) / E\left(C_{i}\right) J(R)$ is not isomorphic to $E\left(C_{i^{\prime}}\right) / E\left(C_{i^{\prime}}\right) J(R)$ if $i \neq i^{\prime}$. Thus, each simple right module has a projective cover and this means that $R$ is semiperfect by [1, Theorem 27.6]. Therefore, $R$ is semilocal.

$(4) \Rightarrow(1)$. We first show that $\operatorname{Soc}\left(R_{R}\right)$ is finitely generated. As we know that $R$ is semilocal, we may write $R / J=\oplus_{i=1}^{n} D_{i}$ with each $D_{i}$, a simple module. Assume that $\operatorname{Soc}\left(R_{R}\right)$ is not finitely generated and choose a direct sum $\oplus_{k=1}^{n+1} C_{k}$ of simple modules in $\operatorname{Soc}\left(R_{R}\right)$. By hypothesis, $\oplus_{k=1}^{n+1} E\left(C_{k}\right)$ is a finitely generated submodule of $E\left(R_{R}\right)$ and thus, it embeds in $R$. This means that there exists a set $\left\{e_{k} \mid k=1, \ldots, n+1\right\}$ of nonzero orthogonal idempotents in $R$ such that $E\left(C_{k}\right)=e_{k} R$ for each $k=1, \ldots, n+1$. But this means that $\oplus_{k=1}^{n+1} e_{k} R / e_{k} J$ is a direct sum of $n+1$ nonzero submodules of $R / J$. A contradiction, since $R / J$ is a semisimple ring of length $n$.

Now we claim that $\operatorname{Soc}\left(R_{R}\right)$ is essential in $R$. We know that $\operatorname{Soc}\left(R_{R}\right)=\oplus_{k=1}^{n} C_{k}$ is finitely generated. So $\oplus_{k=1}^{n} E\left(C_{k}\right)$ is also finitely generated by hypothesis and it embeds in $R$. Let $e_{k} \in R$ be an idempotent such that $E\left(C_{k}\right)=e_{k} R$ for each $k$. Assume that $\oplus_{k=1}^{n} E\left(C_{k}\right)$ is not essential in $R$ and call $e=1-\sum_{k=1}^{n} e_{k}$. Then $R / J=\left(\oplus_{k=1}^{n} e_{k} R / e_{k} J\right) \oplus e R / e J$. Again a contradiction since the length of $R / J$ is $n$.

Therefore, $R=\oplus_{k=1}^{n} e_{k} R \cong \oplus_{k=1}^{n} E\left(C_{k}\right)$ is a right self-injective ring and thus $R / J=\oplus_{k=1}^{n} e_{k} R / e_{k} R$. Let us note that if $e_{k} R / e_{k} J \varsubsetneqq e_{k^{\prime}} R / e_{k^{\prime}} J$, then $e_{k} R \varsubsetneqq e_{k^{\prime}} R$ either, as they are their projective covers. And thus, $C_{k} ¥ C_{k^{\prime}}$. This means that $R$ must contain all isomorphism classes of simple right $R$-modules and so, it is a right cogenerator.

Motivated by the above theorem, we would like to propose the following conjecture.

Conjecture 2.7. If $R$ is a right cogenerator right $R$-tight ring, then $R$ is right $P F$.

We are now going to obtain several corollaries of Theorem 2.6 which will give partial answers to the above proposed conjecture. Recall that a ring $R$ is called right automorphism-invariant if it is invariant under any automorphism of its injective envelope $E\left(R_{R}\right)$ (see e.g. [15]).

Corollary 2.8. Let $R$ be a right $R$-tight, right automorphism-invariant ring such that $R_{R}$ is a cogenerator. Then $R$ is right $P F$.

Proof. It is shown in [15] that $R$ is semiregular and $Z\left(R_{R}\right)$ is the Jacobson radical of $R$. Therefore, $R / Z\left(R_{R}\right)$ is von Neumann regular and this means that the Jacobson radical of $R / Z\left(R_{R}\right)$ is completely nil. The result now follows from Theorem 2.6 
It is clear that if $R$ is a right extending ring, then every cyclic submodule of $E\left(R_{R}\right)$ essentially embeds in a projective module if and only if $R_{R}$ is $R$-tight. Therefore, we have:

Corollary 2.9. Let $R$ be a ring. Then the following conditions are equivalent:

(1) $R$ is right $P F$.

(2) $R_{R}$ is $R$-tight, extending and a cogenerator.

(3) $R_{R}$ is $R$-tight, extending and the injective envelopes of simple right $R$ modules are projective.

Proof. The implications $(1) \Rightarrow(2) \Rightarrow(3)$ are clear.

$(3) \Rightarrow(1)$ Clearly $R_{R}$ is a cogenerator. Using the results of [11] it is possible to show that every cyclic submodule of $E\left(R_{R}\right)$ essentially embeds in a projective module, for if $R_{R}$ is $R$-tight and extending, then every cyclic submodule of $E\left(R_{R}\right)$ is essentially embeddable in a projective module and so we may apply [11, Theorem 3.1]. The implication now follows from Theorem 2.6

Our first example shows that we cannot drop from Theorem 2.6 and Corollary 2.9 the hypothesis that $R_{R}$ is a cogenerator.

Example 2.10. The ring of rational integers $\mathbf{Z}$ is both tight and extending but it is not self-injective. Therefore, it is not PF.

The next example shows that a right $R$-tight ring does not need to be right extending.

Example 2.11. The ring $R$ of upper triangular matrices over a field $F$ is right $R$-tight. Furthermore, since $E\left(R_{R}\right)$ is projective, every direct summand of $E\left(R_{R}\right)$ has an essential finitely generated projective submodule. However, $R$ is not a right extending ring, for the right ideal $\left\{\left(\begin{array}{ll}0 & x \\ 0 & x\end{array}\right) \mid x \in F\right\}$ is not essential in a direct summand of $R_{R}$.

Note that the ring constructed in the above example is not right tight since otherwise it would be quasi-Frobenius as it is right artinian. Next, we give example of a ring $R$ such that every finitely generated submodule of its injective envelope $E\left(R_{R}\right)$ embeds in a free module but $R$ is not right extending.

Example 2.12. Let $R$ be a right noetherian ring such that the injective envelope of any flat module is flat. For instance, a commutative noetherian domain (see [6. Theorem 3]). As this property is clearly Morita invariant, any flat right module over $M_{n}(R)$ has a flat injective envelope for any $n \geq 1$. Let $E=E\left(M_{n}(R)_{M_{n}(R)}\right)$ and let $p: M_{n}(R)^{(I)} \rightarrow E$ be an epimorphism. As $E$ is flat, $p$ is a pure epimorphism. Let $N$ be any finitely generated submodule of $E$. As $R$ is right noetherian, $N$ is finitely presented and thus, the inclusion $i: N \rightarrow E$ lifts to a monomorphism $v: N \rightarrow M_{n}(R)^{(I)}$. Therefore, $M_{n}(R)$ is a ring such that every finitely generated submodule of its right injective envelope embeds in a free module. However, if $R$ is a commutative noetherian domain which is not semihereditary, then $M_{n}(R)$ is not right (nor left) extending (see [3, Example 2.3.13]).

Finally, we exhibit an example of a commutative ring $R$ which is tight, but it does not have the property that every direct summand of $E\left(R_{R}\right)$ has an essential finitely generated projective submodule, nor every cyclic submodule of $E\left(R_{R}\right)$ is essentially embeddable in a projective. In particular, $R$ is not extending. 
Example 2.13. Let $R=\{(m, n) \in \mathbf{Z} \times \mathbf{Z} \mid m \equiv n(\bmod 2)\} \subseteq \mathbf{Z} \times \mathbf{Z}$. Then $R$ is a semiprime Goldie ring and, in fact, $R$ is an order in the semisimple ring $\mathbf{Q} \times \mathbf{Q}$, so that $E\left(R_{R}\right) \cong \mathbf{Q} \times \mathbf{Q}$. Using [17, Proposition 4.2] one can easily see that as $R$ is a semiprime (two-sided) Goldie ring, then $R_{R}$ is tight, cf. [19].

On the other hand, the principal ideal $K_{R}=R(2,0)=2 \mathbf{Z} \times 0 \subseteq R$ is an essential submodule of $\mathbf{Q} \times 0=\{(q, 0) \mid q \in \mathbf{Q}\}$ and, since the latter module is divisible, it is injective and hence $E\left(K_{R}\right) \cong \mathbf{Q} \times 0$. Assume then that $K_{R}$ is essentially embeddable in a projective module $P_{R}$. Then $P_{R}$ embeds in $\mathbf{Q} \times 0$. On the other hand, if we set $L=R(0,2) \subseteq R$, then it is easily checked that $R / L \cong \mathbf{Z}$ and that $P=P / L P$ is also a projective $R / L$-module. Thus $P$ can be viewed as a $\mathbf{Z}$-projective submodule of $\mathbf{Q} \times 0$ and this implies that $P$ is cyclic as $R / L$-module and hence as $R$-module. Thus there exists $0 \neq(q, 0) \in \mathbf{Q} \times 0$ such that $P=R(q, 0)$. The map $K \rightarrow P$ defined by $(2 n, 0) \mapsto(q n, 0)$ is easily seen to be an isomorphism, and so we must have $P_{R} \cong K_{R}$. But, since $R_{R}$ is indecomposable, it is clear that $K_{R}$ is not projective, which gives a contradiction and shows that $K_{R}$ is not essentially embeddable in a projective module. Observe also that, in particular, $R$ cannot be a extending ring.

It is well known that a ring $R$ is right $\mathrm{PF}$ if and only if it is right self-injective and has finite essential socle (i.e., $R_{R}$ is finitely cogenerated). The following result extends this fact.

Proposition 2.14. Let $R$ be a ring such that $R_{R}$ is tight and $E\left(R_{R}\right)$ is both finitely generated and finitely cogenerated. Then $R$ is right $P F$.

Proof. Since $R_{R}$ is tight and $E\left(R_{R}\right)$ is finitely generated, $E\left(R_{R}\right)$ embeds in $R_{R}$ and so there exists $X \subseteq R_{R}$ such that $R_{R} \cong E\left(R_{R}\right) \oplus X$. Then $\operatorname{Soc}\left(R_{R}\right) \cong$ $\operatorname{Soc}\left(E\left(R_{R}\right)\right) \oplus \operatorname{Soc}(X) \cong \operatorname{Soc}\left(R_{R}\right) \oplus \operatorname{Soc}(X)$ (since $\operatorname{Soc}\left(R_{R}\right)$ is essential in $\left.R_{R}\right)$. Now, since $\operatorname{Soc}\left(R_{R}\right)$ is finitely generated, we see by Krull-Remak-Schmidt that $\operatorname{Soc}(X)=0$. Since $\operatorname{Soc}\left(R_{R}\right)$ is essential in $R_{R}$, this implies that $X=0$ and so $R$ is right self-injective and hence right $\mathrm{PF}$ by [23, 12.5.2].

Recall that a module $M$ is said to be finite dimensional if it does not contain an infinite direct sum of nonzero submodules.

Theorem 2.15. Let $R$ be a ring such that each indecomposable injective right $R$ module is projective and every projective right $R$-module is $R$-tight. Then $R$ is a QF ring.

Proof. By [19, Theorem 2.6], since each direct sum of indecomposable injective modules is projective and hence $R$-tight, $R$ has the property that each finitely generated right $R$-module is finite dimensional. Then, if $X$ is a finitely generated right $R$-module, $X$ contains an essential submodule of the form $\oplus_{i=1}^{n} U_{i}$, where the $U_{i}$ 's are uniform modules. Thus $E(C) \cong \oplus_{i=1}^{n} E\left(U_{i}\right)$ is a finite direct sum of indecomposable injective modules and thus, projective. Each $E\left(C_{i}\right)$ has a local endomorphism ring and hence it is a projective local module and, in particular, cyclic. Thus, we see that $X$ is essentially embeddable in a finitely generated projective module and by Corollary 2.2, $R$ is QF.

Remark 2.16. Observe that the hypothesis of the above theorem is weaker than in [20, Theorem 5.1], since the assumption that $R$ is semiperfect (together with the other things in hypothesis) implies that every indecomposable injective is projective.

Our next proposition is a simple yet useful observation. 
Proposition 2.17. Any left perfect and right tight ring is right self-injective.

Proof. Assume that, on the contrary, $R \neq E\left(R_{R}\right)$, so that there exists $x \in E\left(R_{R}\right)$ such that $x \notin R$. Then $R_{R}+x R$ is a finitely generated submodule of $E\left(R_{R}\right)$ and, as $R$ is right tight, there is an embedding $R_{R}+x R \subseteq R_{R}$. But, since $R_{R}$ embeds, as a proper submodule, in $R_{R}+x R$, we get a proper embedding of $R_{R}$ into itself, which gives an infinite descending chain of principal right ideals of $R$, a contradiction.

Corollary 2.18. If $R$ is right artinian and right tight, then $R$ is $Q F$.

Remark 2.19. Note that, however, a right noetherian, right extending and right tight ring does not need to be $Q F$, even in the commutative case, as the example $R=\mathbf{Z}$ shows.

\section{An extension of the FGF conjecture.}

We have studied in the above section different new characterizations of $\mathrm{PF}$ rings in terms of $(R$-)tight conditions and we have outlined the strong relation existing between these characterizations and both CF and FGF conjectures. Our purpose in this section is to establish a general problem such that all the existing partial results on $\mathrm{CF}$ and FGF conjectures can be included as partial positive answers of this new problem. In order to do so, we are going to define that a ring $R$ is generalized right $(R$-)tight if every finitely generated (resp., cyclic) submodule of $E\left(R_{R}\right)$ embeds in a free module. The results in the above section naturally suggest to propose the following conjecture:

Conjecture 3.1. If $R$ is a right Kasch right generalized (R-)tight ring, then $R_{R}$ has finitely generated essential socle.

We would like to remark that Conjecture 2.7 is a consequence of this other conjecture, since if Conjecture 3.1 is true, then every right cogenerator right $R$-tight ring has finitely generated essential right socle. One can then use the same arguments as in Theorem 2.6 to show that $R$ is right PF. On the other hand, Theorem 2.1 shows that Conjecture 3.1 is true if we assume that every cyclic submodule of $E\left(R_{R}\right)$ essentially embeds in a projective module or that $R$ is right extending. Moreover, Osofsky's pioneering characterization of right PF rings [28, Theorem 1] is a positive solution to the conjecture when $R$ is right self injective. Indeed, it is not difficult to check that most additional conditions which are known to force a right $\mathrm{CF}$ ring to be right artinian, also force the above conjecture to be true. We are going to show examples of them.

Proposition 3.2. Let $R$ be a right Kasch right generalized $R$-tight ring. If $R_{R}$ is noetherian, then $\operatorname{Soc}\left(R_{R}\right)$ is finitely generated and essential in $R$.

Proof. Assume that $R$ is a right generalized right $R$-tight ring. Then every right ideal is a right annihilator. The result now follows from [9, 3.5B Johns' Lemma] (see also [27, Theorem 8.9]).

Proposition 3.3. Let $R$ be a right Kasch right generalized tight ring. If $R$ is also left Kasch, then $\operatorname{Soc}\left(R_{R}\right)$ is finitely generated and essential in $R$. In particular, any commutative Kasch generalized tight ring has finitely generated essential socle.

Proof. Note that, as $R_{R}$ is Kasch and generalized tight, any finitely generated right module embeds in a free module. And, as ${ }_{R} R$ is also Kasch, $R$ is an S-ring in the sense of [24]. The result now follows from [24, Theorem 1, (3) $\Rightarrow(1)]$. 
One may note that, the assumption that $R_{R}$ is $R$-tight is critical in the proof of Theorem 2.5. We do not know if this theorem is still valid under the weaker assumption that $R_{R}$ is generalized $R$-tight, and thus give a positive solution to Conjecture 3.1 when the Jacobson radical of $R / Z\left(R_{R}\right)$ is completely nil. However, our next theorem shows that the arguments can be adapted if we assume the slightly stronger assumption that the Jacobson radical of $R / Z\left(R_{R}\right)$ is completely right Tnilpotent. Recall that an ideal $I$ of a ring $R$ is called right T-nilpotent if for any infinite sequence $r_{1}, \ldots, r_{n}, \ldots$ of elements in $I$, there exists an $n_{0} \geq 1$ such that $r_{n_{0}} \cdot \ldots \cdot r_{1}=0$ (see [30, p. 183, Definition before Proposition 2.5]). And we are going to say that the Jacobson radical of a ring $S$ is completely right T-nilpotent if every ring $S^{\prime}$ which is a homomorphic image of $S$ has right T-nilpotent Jacobson radical.

Theorem 3.4. Let $R$ be a right Kasch ring such that any cyclic submodule of $E\left(R_{R}\right)$ embeds in a free module. If the Jacobson radical of $R / Z\left(R_{R}\right)$ is completely right T-nilpotent, then $\operatorname{Soc}\left(R_{R}\right)$ is finitely generated and essential in $R_{R}$.

Proof. The general scheme of the proof is quite similar to that of Theorem 2.5 Therefore, we will only elaborate on the part where the proof is different and otherwise we will just refer to the relevant part of the proof of Theorem 2.5. We will follow the same notations as in Theorem 2.5.

Step 1. We claim that $\operatorname{Soc}\left(R_{R}\right)$ contains only finitely many homogeneous components.

Let us assume on the contrary that $\operatorname{Soc}\left(R_{R}\right)$ has infinitely many homogeneous components and let $\left\{C_{i}\right\}_{i \in I}$ be a representative set of the isomorphism classes of simple modules in $\operatorname{Soc}\left(R_{R}\right)$. Proceeding exactly as in the proof of Theorem 2.5. we get $\left\{e_{K}+\mathcal{N} \mid K \in \mathcal{K}\right\}$, an orthogonal family of nonzero central idempotents in $S / \mathcal{N}$.

Let $u: R \rightarrow E$ be the inclusion of $R_{R}$ in its injective envelope and call $x_{K, 1}=$ $e_{K} \circ u(1) \in E$. Then $e_{K} \circ u$ factors as

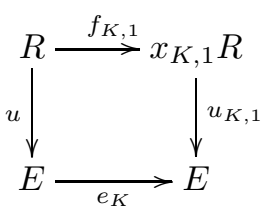

where $f_{K, 1}$ is an epimorphism and $u_{K, 1}$, a monomorphism. As we are assuming that any cyclic submodule of $E\left(R_{R}\right)$ embeds in a free module (of finite rank), there exists a monomorphism $\alpha_{K, 1}: x_{K, 1} R \rightarrow R^{\left(n_{K, 1}\right)}$, for some $n_{K, 1} \geq 1$, that extends by injectivity to an $s_{K, 1}: E \rightarrow E^{\left(n_{K, 1}\right)}$ such that $u^{\left(n_{K, 1}\right)} \circ \alpha_{K, 1}=s_{K, 1} \circ u_{K, 1}$, where we are denoting by $u^{\left(n_{K, 1}\right)}: R^{\left(n_{K, 1}\right)} \rightarrow E^{\left(n_{K, 1}\right)}$ the inclusion. Again, as $\left.s_{K, 1}\right|_{E\left(x_{K, 1} R\right)}: E\left(x_{K, 1} R\right) \rightarrow E$ is injective, there exists an $h_{K, 1}: E^{\left(n_{K, 1}\right)} \rightarrow E$ such that $h_{K, 1} \circ s_{K, 1} \circ e_{K}=e_{K}$

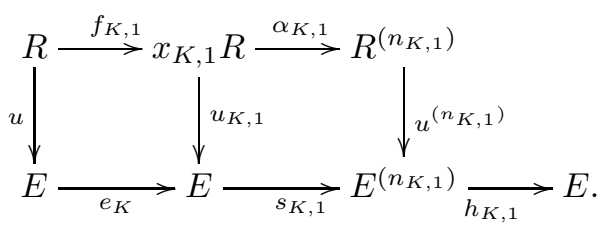


Let $\pi_{K, 1, t}: E^{\left(n_{K, 1}\right)} \rightarrow E$ and $v_{K, 1, t}: E \rightarrow E^{\left(n_{K, 1}\right)}$, for $t=1, \ldots, n_{K, 1}$ be the canonical projections and injections. Then $\sum_{t=1}^{n_{K, 1}} v_{K, 1, t} \circ \pi_{K, 1, t}=1_{E^{\left(n_{K, 1}\right)}}$. Therefore, we have that

$$
\begin{aligned}
e_{K} & =h_{K, 1} \circ s_{K, 1} \circ e_{K} \\
& =h_{K, 1} \circ\left(\sum_{t=1}^{n_{K, 1}} v_{K, 1, t} \circ \pi_{K, 1, t}\right) \circ s_{K, 1} \circ e_{K} \\
& =\sum_{t=1}^{n_{K, 1}}\left(h_{K, 1,} \circ v_{K, 1, t} \circ \pi_{K, 1, t} \circ s_{K, 1} \circ e_{K}\right)
\end{aligned}
$$

As $e_{K}+J \notin \mathcal{N} / J$, there exists a $t_{0}$ such that

$$
\left(h_{K, 1} \circ v_{K, 1, t_{0}} \circ \pi_{K, 1, t_{0}} \circ s_{K, 1} \circ e_{K}\right)+J \notin \mathcal{N} / J .
$$

Call $s_{K, 1}^{\prime}=\pi_{K, 1, t_{0}} \circ s_{K, 1}, h_{K, 1}^{\prime}=h_{K, 1} \circ v_{K, 1, t_{0}}$ and $r_{K, 1}=s_{K, 1}^{\prime} \circ e_{K} \circ u(1) \in R$. And let $x_{K, 2}=h_{K, 1}^{\prime} \circ u\left(r_{K, 1}\right)=h_{K, 1}^{\prime} \circ s_{K, 1}^{\prime} \circ e_{K} \circ u(1) \in E$. Again, as any cyclic submodule of $E\left(R_{R}\right)$ embeds in a free module, there exists a monomorphism $\alpha_{K, 2}: x_{K, 2} R \rightarrow R^{\left(n_{K, 2}\right)}$, for some $n_{K, 2} \geq 1$, that extends by injectivity to an $s_{K, 2}: E \rightarrow E^{\left(n_{K, 2}\right)}$ such that $u^{\left(n_{K, 2}\right)} \circ \alpha_{K, 2}=s_{K, 2} \circ u_{K, 2}$. And, as $\left.s_{K, 2}\right|_{E\left(x_{K, 2} R\right)}$ : $E\left(x_{K, 2} R\right) \rightarrow E^{\left(n_{K, 2}\right)}$ is injective, there exists an $h_{K, 2}: E^{\left(n_{K, 2}\right)} \rightarrow E$ such that $\left.h_{K, 2} \circ s_{K, 2}\right|_{E\left(x_{K, 2} R\right)}=\left.1\right|_{E\left(x_{K, 2} R\right)}$. Moreover, as by construction,

$$
h_{K, 2} \circ s_{K, 2} \circ h_{K, 1}^{\prime} \circ s_{K, 1}^{\prime} \circ e_{K} \circ u=h_{K, 1}^{\prime} \circ s_{K, 1}^{\prime} \circ e_{K} \circ u
$$

we get that $h_{K, 2} \circ s_{K, 2} \circ h_{K, 1}^{\prime} \circ s_{K, 1}^{\prime} \circ e_{K}-h_{K, 1}^{\prime} \circ s_{K, 1}^{\prime} \circ e_{K} \in J(S)$. Therefore, $h_{K, 2} \circ s_{K, 2} \circ h_{K, 1}^{\prime} \circ s_{K, 1}^{\prime} \circ e_{K}+J \notin \mathcal{N} / J$, as neither is $h_{K, 1}^{\prime} \circ s_{K, 1}^{\prime} \circ e_{K}+J \notin \mathcal{N} / J$.

Let $\pi_{K, 2, t}: E^{\left(n_{K, 2}\right)} \rightarrow E$ and $v_{K, 2, t}: E \rightarrow E^{\left(n_{K, 2}\right)}$, for $t=1, \ldots, n_{K, 2}$ be the canonical projections and injections. As before, there exists a $t_{0}$ such that such that $R$.

$$
\left(h_{K, 2} \circ v_{K, 2, t_{0}} \circ \pi_{K, 2, t_{0}} \circ s_{K, 2} \circ h_{K, 1}^{\prime} \circ s_{K, 1}^{\prime} \circ e_{K}\right)+J \notin \mathcal{N} / J .
$$

Call now $s_{K, 2}^{\prime}=\pi_{K, 2, t_{0}} \circ s_{K, 2}, h_{K, 2}^{\prime}=h_{K, 2} \circ v_{K, 2, t_{0}}$ and $r_{K, 2}=s_{K, 2}^{\prime} \circ h_{K, 1} \circ u(1) \in$

Repeating the same arguments, we can define $s_{K, l}^{\prime}, h_{K, l}^{\prime} \in S$ and $r_{K, l} \in R$, for each $l \geq 1$ such that:

- $\left(h_{K, l}^{\prime} \circ s_{K, l}^{\prime} \circ \ldots \circ h_{K, 1}^{\prime} \circ s_{K, 1}^{\prime} \circ e_{K}\right)+J \notin \mathcal{N} / J$ for any $l \geq 1$.

- $\Psi\left(r_{K, 1}+\mathcal{M}\right)=s_{K, 1}^{\prime} \circ e_{K}+\mathcal{N}$.

- $\Psi\left(r_{K, l+1}+\mathcal{M}\right)=s_{K, l+1}^{\prime} \circ h_{K, l}^{\prime}+\mathcal{N}$ for each $l \geq 1$.

We claim that there exists some $l \geq 1$ such that $r_{K, l}+\mathcal{M} \notin J(R / \mathcal{M})$. Assume on the contrary that $r_{K, l}+\mathcal{M} \in J(R / \mathcal{M})$ for each $l \geq 1$. As we are assuming that the Jacobson radical of $R / Z\left(R_{R}\right)$ is completely right T-nilpotent, there exists an $l_{0}$ such that $r_{K, l_{0}+1} \cdot \ldots \cdot r_{K, 1}+\mathcal{M}=0$ in $R / \mathcal{M}$. But this means that

$$
\left(s_{K, l_{0}+1}^{\prime} \circ h_{K, l_{0}}^{\prime} \circ \ldots \circ s_{K, 1}^{\prime} \circ e_{K}\right)+\mathcal{N}=\Psi\left(\left(r_{K, l_{0}+1} \cdot \ldots \cdot r_{K, 1}+\mathcal{M}\right)\right)=0
$$

in $S / \mathcal{N}$, a contradiction. This proves our claim.

Call $r_{K}=r_{K, l_{0}}, s_{K}^{\prime}=s_{K, l_{0}+1}^{\prime}$ and $h_{K}^{\prime}=h_{K, l_{0}}^{\prime}$. As $e_{K}+J$ is central in $S / J$, we deduce that $s_{K}^{\prime} \circ h_{K}^{\prime} \circ e_{K}+J \notin S / \mathcal{N}$. On the other hand, as $r_{K}+\mathcal{M} \notin J(R / \mathcal{M})$, there exists a maximal right ideal $L_{K} / \mathcal{M}$ of $R / \mathcal{M}$ such that $r_{K}+\mathcal{M} \notin L_{K} / \mathcal{M}$ and thus, $R / L_{K}$, is a simple right $R / \mathcal{M}$-module such that $R / L_{K} \cdot\left(r_{K}+\mathcal{M}\right) \neq 0$.

Now as in the proof of Theorem 2.5, we show that $e_{K} \circ e_{K^{\prime}} \notin \mathcal{N}$. But this is a contradiction, since $e_{K} \circ e_{K^{\prime}} \in \mathcal{N}$ by construction.

We have constructed then $|K|$ isomorphism classes of simple right $R$-modules. This yields a contradiction, since $|\mathcal{K}| \gtreqless|I|$. 
Step 2. We claim that any homogeneous component of $\operatorname{Soc}\left(R_{R}\right)$ is finitely generated.

Assume on the contrary that there exists a homogeneous component which is not finitely generated. We already know that there are only finitely many homogeneous components in $\operatorname{Soc}\left(R_{R}\right)$. Let $\left\{C_{1}, \ldots, C_{m}\right\}$ be a representative set of simple modules belonging to them.

Let us fix a simple module $C_{i}$, for $i=1, \ldots, m$, and call $C_{i, 1}=C_{i}$. Let $e_{i, 1} \in S$ be an idempotent such that $E\left(C_{i, 1}\right)=e_{i, 1} E$ and let us repeat the reasonings made in Step 1, in order to decompose $e_{i, 1} \circ u=u_{i, 1} \circ f_{i, 1}$, where $f_{i, 1}: R \rightarrow x_{i, 1} R$ is an epimorphism and $u_{i, 1}: x_{i, 1} R \rightarrow E$, a monomorphism. As each cyclic submodule of $E\left(R_{R}\right)$ embeds in a free module, there exists a monomorphism $\alpha: x_{i, 1} R \rightarrow R^{n}$, for some $n \geq 1$. So there exists a projection $\pi: R^{n} \rightarrow R$ such that $\left.\pi \circ \alpha\right|_{C_{i, 1}} \neq 0$. And, as the simple module $C_{i, 1}$ is essential in $x_{i, 1} R$, we deduce that $\pi \circ \alpha$ is a monomorphism. Call it $\alpha_{i, 1}$. This monomorphism extends by injectivity to an endomorphism $s_{i, 1}: E \rightarrow E$ such that $s_{i, 1} \circ u_{i, 1}=u \circ \alpha_{i, 1}$. Note that $s_{i, 1} \circ e_{i, 1} \notin J(S)$ since $\left.s_{i, 1} \circ e_{i, 1}\right|_{C_{i, 1}}$ is a monomorphism and thus, $s_{i, 1} \circ e_{i, 1}$ does not have essential kernel.

Let us call $C_{i, 2}=s_{i, 1} \circ e_{i, 1}\left(C_{i, 1}\right)$. Then $C_{i, 2}$ is a simple right $R$-module isomorphic to $C_{i, 1}$ and therefore, we can repeat the above construction to obtain $e_{i, 2}, s_{i, 2} \in S$ such that $\left.\left(s_{i, 2} \circ e_{i, 2}\right)\right|_{C_{i, 2}}$ is injective and therefore, $\left.\left(s_{i, 2} \circ e_{i, 2} \circ s_{i, 1} \circ e_{i, 1}\right)\right|_{C_{i, 1}}$ is also injective. So $s_{i, 2} \circ e_{i, 2} \circ s_{i, 1} \circ e_{i, 1} \notin J(S)$. Repeating this construction, we can find by recurrence idempotents $e_{i, n} \in S$ and elements $s_{i, n} \in S$ such that $\left(s_{i, n} \circ e_{i, n} \circ \ldots \circ\right.$ $\left.s_{i, 1} \circ e_{i, 1}\right)\left.\right|_{C_{i}, 1}$ is a monomorphism, and therefore, $s_{i, n} e_{i, n} \circ \ldots \circ s_{i, 1} \circ e_{i, 1} \notin J(S)$.

Let $r_{i, n}=s_{i, n} \circ e_{i, n} \circ u(1) \in R$ for each $n \geq 1$. We claim that $r_{i, n}+Z\left(R_{R}\right) \notin$ $J\left(R / Z\left(R_{R}\right)\right)$ for some $n \geq 1$. Assume on the contrary that $r_{i, n}+Z\left(R_{R}\right) \in$ $J\left(R / Z\left(R_{R}\right)\right)$ for every $n \geq 1$. As we are assuming that $J\left(R / Z\left(R_{R}\right)\right)$ is completely right T-nilpotent, there exists an $n_{0}$ such that $r_{i, n_{0}} \cdot \ldots \cdot r_{i, 1}+Z\left(R_{R}\right)=0$ in $R / Z\left(R_{R}\right)$. Thus, $\Phi\left(r_{i, n_{0}} \ldots r_{i, 1}+Z\left(R_{R}\right)\right)=0$ in $S / J$. But, as by construction, $\Phi\left(r_{i, n}+Z\left(R_{R}\right)\right)=s_{i, n} \circ e_{i, n}+J$, we deduce that $\left(s_{i, n_{0}} \circ e_{i, n_{0}} \circ \ldots \circ s_{i, 1} \circ e_{i, 1}\right) \in J(S)$, a contradiction. This proves our claim.

Let us choose an $n$ such that $r_{i, n}+Z\left(R_{R}\right) \notin J\left(R / Z\left(R_{R}\right)\right)$ and set $r_{i}=r_{i, n}, e_{i}=$ $e_{i, n}, s_{i}=s_{i, n}$. Replacing, if necessary $C_{i}$ by its isomorphic image $C_{i, n}$, we get that $E\left(C_{i}\right)=e_{i} E$. Moreover, as $r_{i}+Z\left(R_{R}\right) \notin J\left(R / Z\left(R_{R}\right)\right)$, there exists a maximal right ideal $L_{i} / Z\left(R_{R}\right)$ of $R / Z\left(R_{R}\right)$ such that $r_{i}+Z\left(R_{R}\right) \notin L_{i} / Z\left(R_{R}\right)$. Call $D_{i}=R / L_{i}$. Then $D_{i}$ is a simple $R / Z\left(R_{R}\right)$-module such that $D_{i} \cdot\left(r_{i}+Z\left(R_{R}\right)\right) \neq 0$.

We claim that $D_{i} \not D_{j}$ if $i \neq j$. Assume on the contrary that $\delta: D_{i} \rightarrow D_{j}$ is an isomorphism. As $D_{i} \cdot\left(r_{i}+Z\left(R_{R}\right)\right) \neq 0$, we can choose an $0 \neq x \in D_{i}$ such that $x \cdot\left(r_{i}+Z\left(R_{R}\right)\right) \neq 0$ and thus, $\delta(x) \cdot\left(r_{i}+Z\left(R_{R}\right)\right)$ is a generator of $D_{j}$. Again, as $D_{j} \cdot\left(r_{j}+Z\left(R_{R}\right)\right) \neq 0$, there exists an $r \in R$ such that $\delta(x)\left(r_{i} r r_{j}+Z\left(R_{R}\right)\right) \neq 0$. In particular, $r_{i} r r_{j} \notin Z\left(R_{R}\right)$. And thus,

$$
\left(s_{i} \circ e_{i}+J\right) \circ \Phi\left(r+Z\left(R_{R}\right)\right) \circ\left(s_{j} \circ e_{j}+J\right)=\Phi\left(r_{i} r r_{j}+Z\left(R_{R}\right)\right) \neq 0 .
$$

Let

$$
g=\left(s_{i}+J\right) \circ\left(e_{i}+J\right) \circ \Phi\left(r+Z\left(R_{R}\right)\right) \circ\left(s_{j}+J\right): S / J \longrightarrow S / J .
$$

Then, as $\operatorname{Im}\left(\left(e_{i}+J\right) \circ \Phi\left(r+Z\left(R_{R}\right)\right) \circ\left(s_{j}+J\right)\right)$ is contained in the simple right ideal $\left(e_{i} S+J\right) / J$, we deduce that $\operatorname{Im}(g)$ is contained in a simple right ideal of $S / J$, say $Y$, isomorphic to $\left(e_{i} S+J\right) / J$. But then, as $\left(s_{i} \circ e_{i}+J\right) \circ \Phi\left(r+Z\left(R_{R}\right)\right) \circ\left(s_{j} \circ e_{j}+J\right) \neq 0$, we deduce that the homomorphism $\left(s_{j}+J\right) \circ\left(e_{j}+J\right) \circ \Phi\left(r+Z\left(R_{R}\right)\right) \circ\left(s_{i}+J\right)$ 
is not zero either, when restricted to the simple right ideal $\left(e_{j} S+J\right) / J$. And this means that $\left(e_{j} S+J\right) / J \cong Y \cong\left(e_{i} S+J\right) / J$. Therefore, $e_{i} S \cong e_{j} S$, since they are the projective covers of $\left(e_{i} S+J\right) / J$ and $\left(e_{j} S+J\right) / J$, respectively. But this means that $E\left(C_{i}\right) \cong E\left(C_{j}\right)$ and so, $C_{i} \cong C_{j}$, a contradiction. This proves our claim.

Lets now assume that some homogeneous component is not finitely generated. Say that it is the homogeneous component associated to $C_{1}$. Then as in the proof of Theorem 2.5. we arrive at a contradiction. This shows that each homogeneous component of $\operatorname{Soc}\left(R_{R}\right)$ is finitely generated and thus, $\operatorname{Soc}\left(R_{R}\right)$ is finitely generated.

Step 3. We finally claim that $\operatorname{Soc}\left(R_{R}\right)$ is essential in $R_{R}$. The proof of this part is identical to the step 3 of Theorem 2.5 and this completes the proof.

We can now state the following partial answer to the CF and FGF conjectures.

Corollary 3.5. Let $R$ be a right $C F$ ring. If the Jacobson radical of $R / Z\left(R_{R}\right)$ is completely right $T$-nilpotent, then $R$ is right artinian. In particular, if $R$ is right $F G F$, then it is $Q F$.

Proof. By Theorem 3.4, we know that $R$ has a finitely generated essential right socle and thus, any cyclic right $R$-module also has a finitely generated essential socle, since it embeds in a (finitely generated) free module. Therefore, $R$ is right artinian (see e.g. [1, Theorem 10.4] and [1, Proposition 10.10]).

Our next corollary shows that the main result of 14 is a consequence of our Corollary 3.5.

Corollary 3.6. Let $R$ be a ring and assume that $R / Z\left(R_{R}\right)$ is a von Neumann regular ring. Then:

(1) If $R$ is right $C F$, then it is right artinian.

(2) If $R$ is right $F G F$, then it is $Q F$.

Proof. If $R / Z\left(R_{R}\right)$ is von Neumann regular, then every ring which is a homomorphic image of $R / Z\left(R_{R}\right)$ has zero Jacobson radical. We may now apply Corollary 3.5 .

We close the paper by extending [29, Theorem 2].

Corollary 3.7. Let $R$ be a right $C F$ ring such that $R / J(R)$ is von Neumann regular and $J(R)$ is right T-nilpotent. Then $R$ is right artinian. In particular, if $R$ is right $F G F$, then it is $Q F$.

Proof. As $J(R)$ is right T-nilpotent, idempotents lift modulo $J(R)$ (see e.g. 30, Proposition 4.2]). So the ring is semiregular. We claim that $Z\left(R_{R}\right) \subseteq J(R)$. Choose any $r \in Z\left(R_{R}\right)$ and call $f: R \rightarrow R$ the homomorphism given by left multiplication by $r$. As $r R$ is finitely generated, there exists an idempotent $e \in R$ such that $e R \subseteq x R$ and $x R \cap(1-e) R$ is superfluous in $R$ (see [26. Theorem 1.6]). But, as $\operatorname{Ker}(f)$ is essential in $R, e=0$, and this means that $x R=x R \cap(1-e) R$ is superfluous in $R$. So $r \in J(R)$.

Now, let $M / Z\left(R_{R}\right)$ be any two-sided ideal of $R / Z\left(R_{R}\right)$. As $Z\left(R_{R}\right) \subseteq J(R)$, we have a surjective homomorphism of rings $\varphi: R / M \rightarrow R /(M+J)$. And then, $\varphi(J(R / M)) \subseteq J(R /(J+M))$ (see [1, Corollary 15.8]). Therefore, any element 
$r+M \in J(R / M)$ is of the form $j+M$ with $j \in J(R)$. Thus, $J(R / M)$ is right T-nilpotent. The result now follows from Corollary 3.5 .

We close the paper by extending two results of [14].

Corollary 3.8. (see [14, Theorem 16]) Let $R$ be a right CF ring. Then the following conditions are equivalent:

(1) $J\left(R / Z\left(R_{R}\right)\right)$ is completely right T-nilpotent.

(2) Every cyclic right $R$-module essentially embeds in a projective module.

(3) $R_{R}$ is continuous.

Moreover, in this case $Z\left(R_{R}\right)=J(R)$ and $R$ is right artinian.

Proof. (1) $\Rightarrow(2)$ is a consequence of Theorem 3.5 and [14, Corollary 13]. The other implications follow from [14, Theorem 16].

Corollary 3.9. Let $R$ be a ring. Then the following conditions are equivalent:

(1) Every cyclic right $R$-module embeds in $R$ and $J\left(R / Z\left(R_{R}\right)\right)$ is completely right nil.

(2) Every cyclic right $R$-module essentially embeds in a direct summand of $R$.

(3) $R$ is a direct sum of rings which are either right uniserial or finite matrix rings over two-sided uniserial rings.

Proof. (1) $\Rightarrow(2)$ is a consequence of Theorem 2.5 and the proof of 14, Theorem 17]. The other implications follow from [14, Theorem 17].

\section{Acknowledgment.}

Part of this paper was written during a visit of the first author to the Gebze Institute of Technology in Turkey during August 2014 and a visit of the second author to the Department of Mathematics of the University of Murcia in October and November 2014. They would like to thank both departments for their hospitality during their visits.

\section{REFERENCES}

[1] F. W. Anderson and K. R. Fuller, Rings and categories of modules, Springer-Verlag, Berlin and New York, 1974.

[2] G. Azumaya, Completely faithful modules and self-injective rings, Nagoya Math. J. 27 (1966), 697-708.

[3] G. F. Birkenmeier, J. K. Park and, S. T. Rizvi, Extensions of rings and modules, BirkhäuserSpringer, New York, 2013.

[4] J. E. Björk, Rings satisfying a minimum condition on principal ideals, J. Reine Angew. Math. 236 (1969), 112-119.

[5] J. E. Björk, Radical properties of perfect modules, J. Reine Angew. Math. 253 (1972), 78-86.

[6] T. Cheatham and E. Enochs, Injective hulls of flat modules, Comm. Algebra 8, 20 (1980), 1989-1995.

[7] C. W. Curtis and I. Reiner, Representation theory of finite groups and associative algebras, Wiley-Interscience, New York-London, 1962.

[8] F. Dischinger and W. Müller, Left PF is not right PF, Comm. Algebra 14, no. 7 (1986), 1223-1227.

[9] C. Faith, Embedding modules in projectives. A report on a problem, Lecture Notes in Math., vol. 951, Springer-Verlag, Berlin and New York (1982), 21-40.

[10] J. L. Gómez Pardo and P. A. Guil Asensio, Endomorphism rings of completely pure-injective modules, Proc. Amer. Math. Soc. 124 (1996), 2301-2309. 
[11] J. L. Gómez Pardo and P. A. Guil Asensio, Essential embedding of cyclic modules in projectives, Trans. Amer. Math. Soc. 349, 11 (1997), 4343-4353.

[12] J. L. Gómez Pardo and P. A. Guil Asensio, Rings with finite essential socle, Proc. Amer. Math. Soc. 125, 4 (1997), 971-977.

[13] J. L. Gómez Pardo and P. A. Guil Asensio, Embedding in free modules and artinian rings, J. Algebra 198, (1997), 608-617.

[14] J. Gómez Torrecillas and P. A. Guil Asensio, The FGF conjecture and the singular ideal of a ring, J. Algebra Appl. 12, 1350025 (2013) [13 pages] DOI: 10.1142/S0219498813500254.

[15] P. A. Guil Asensio, A. K. Srivastava, Automorphism-invariant modules satisfy the exchange property, J. Algebra 388 (2013), 101-106.

[16] J. S. Golan and S. R. López-Permouth, QI-filters and tight modules, Comm. Algebra 19, 8 (1991), 2217-2229.

[17] S. K. Jain and S. R. López-Permouth, Rings whose cyclics are essentially embeddable in projective modules, J. Algebra 128 (1990), 257-269.

[18] S. K. Jain and S. R. López-Permouth, A generalization of the Wedderburn-Artin Theorem, Proc. Amer. Math. Soc. 106 (1989), 19-23.

[19] S. K. Jain and S. R. López-Permouth, A survey on the theory of weakly injective modules, in Computational Algebra, Lect. Notes Pure Appl. Math. 151, Marcel Dekker, New York (1993), 205-232.

[20] S. K. Jain, S. R. López-Permouth, K. Oshiro and M. A. Saleh, Weakly projective and weakly injective modules, Canad. J. Math. 46 (1994), 971-981.

[21] S. K. Jain, S. R. López-Permouth and M. Saleh, On generalized q.f.d. modules, Arch. Math. (Brno) 41, 3 (2005), 243-251.

[22] S. K. Jain, A. K. Srivastava and A. A. Tuganbaev, Cyclic Modules and the Structure of Rings, Oxford Mathematical Monographs, Oxford Univ. Press, 2012.

[23] F. Kasch, Modules and Rings, Academic Press, London, England, 1982.

[24] T. Kato, Torsionless modules, Tôhoku Math. Journal 20 (1968), 234-243.

[25] T. Y. Lam, Lectures on Modules and Rings, Springer-Verlag, 1999.

[26] W. K. Nicholson, Semiregular modules and rings, Canad. J. Math. 28, no. 5 (1976), 1105-1120.

[27] W. K. Nicholson and M. F. Yousif, Quasi-Frobenius Rings, Cambridge University Press, 2003.

[28] B. L. Osofsky, A generalization of Quasi-Frobenius rings, J. Algebra 4 (1966), 373-387.

[29] M. Saorin and J. P. Rada, On semiregular rings whose finitely generated modules embed in free modules, Canad. Math. Bull. 40 (1997), 221-230.

[30] B. Stenström, Rings of quotients, Springer-Verlag, Berlin and New York, 1975.

[31] A. Tarski, Sur la décomposition des ensembles en sous-ensembles presque disjoints, Fund. Math. 12 (1928), 188-205.

[32] T. S. Tolskaya, When are all cyclic modules essentially embedded in free modules, Mat. Issled. 5 (1970), 187-192.

[33] Y. Utumi, Self-injective rings, J. Algebra 6 (1967), 56-64.

Departamento de Mathematicas, Universidad de Murcia, Murcia, 30100, Spain

E-mail address: paguil@um.es

Gebze Institute of Technology, Department of Mathematics, Kocaeli, 41400 Turkey

E-mail address: ssahinkaya@gyte.edu.tr

Department of Mathematics and Computer Science, St. Louis University, St. Louis, MO-63103, USA

E-mail address: asrivas3@slu.edu 